\title{
Las Defensorías Universitarias como un instrumento para la mejora de las universidades
}

\author{
José Palazón \\ Defensor de la Universidad de Murcia
}

\section{Resumen}

Este artículo recoge mi intervención en el IX Congreso Iberoamericano de Docencia Universitaria, celebrado en Murcia (España) en 2016. Su principal objetivo es presentar el órgano del Defensor Universitario y contribuir, no sólo a su mejor conocimiento, sino a incentivar la implantación de esta figura en las universidades de los países iberoamericanos que todavía no cuentan con ella.

\section{1. ¿Qué es el Defensor Universitario?}

El Defensor Universitario es una figura que existe desde hace décadas en muchas universidades del mundo y tiene por misión fundamental la defensa de los derechos y libertades de los miembros de la comunidad universitaria. En algunos países como España, México o Brasil, el Defensor se ocupa de los derechos de todos los miembros de la comunidad universitaria (profesorado, estudiantes y personal de administración y servicios). Al contrario, en otros países como Austria, Portugal o el Reino Unido, solo puede entender de los derechos de los estudiantes.

En esa función principal de defensa de derechos, hay que resaltar la especial preocupación por la defensa de los derechos de las personas con discapacidad y el trabajo para favorecer su integración en las universidades. Además de esa labor principal, muchos defensores tienen también encomendada la función de hacer propuestas o recomendaciones, incluyendo sugerencias de modificaciones de la normativa universitaria, que puedan contribuir a la mejora de la calidad de la universidad. Las actuaciones del Defensor Universitario también van dirigidas a promover la cultura de la ética en el ámbito universitario.

El Defensor es, en algunos países, una figura que tienen que tener todas las universidades por obligación legal (España, Perú o Portugal). Sin embargo, lo más general es que no exista esa obligación legal y que se trate de una figura creada voluntariamente por algunas universidades (Brasil, Mé- 
xico, Francia o Alemania). También nos podemos encontrar con situaciones como la del Independent Adjudicator que se encarga de la defensa de todos los estudiantes de Inglaterra y Gales o la del Defensor de los Estudiantes en el Ministerio de la Educación Superior de Austria y que son obligatorias legalmente.

\section{Origen de la figura}

Hacia el año 1713 el rey Carlos XII de Suecia creó la oficina del Procurador Supremo, cuya tarea fundamental consistía en supervisar la actuación del conjunto de la administración estatal desde el punto de vista del cumplimiento de la legalidad. Un siglo más tarde, la Constitución de 1809 de ese mismo país crea la figura del Justitieombudsman, independizándolo de la Corona y del Gobierno y convirtiéndolo en un delegado del parlamento encargado de vigilar la observancia de la legalidad por parte de los servidores públicos.

La idea del ombudsman llega a España como una consecuencia más del cambio democrático, incorporándose a nuestro sistema político a través de la Constitución de 1978, y bajo la denominación de Defensor del Pueblo; una figura que también cuenta en nuestro país con algún antecedente como es el caso del Justicia Mayor de Aragón o del Síndic de Greuges. Con la finalidad principal de extraer al máximo todas las potencialidades garantistas inherentes a esta institución, poco a poco se van creando otros ombudsman de ámbito y competencia tanto autonómica como local y sectorial. Se consigue así reforzar -y por la vía de la proximidad y la especialización- los mecanismos de defensa y protección respecto a la vigencia del Estado de derecho consagrado en nuestro ordenamiento constitucional (Rojo Salgado y Gamero Casado, 2012).

Las primeras universidades que implantaron Defensorías fueron norteamericanas. La primera fue la Simon Fraiser University de Canadá, en 1965, y, en Estados Unidos, la primera Defensoría fue la creada en el Montana College en 1966. Esas defensorías se abrieron en un contexto de conflictividad social y, en los Campus, como consecuencia de las protestas por la guerra de Vietnam, acompañando el auge de los movimientos por los derechos civiles. Las primeras Defensorías Universitarias en el ámbito Iberoamericano nacieron hace 30 años en México (UNAM, 1985) y en España, donde, también en 1985, se aprobó la creación de la figura en la Complutense, Granada, León, Barcelona, Extremadura y Salamanca. No obstante, fue la universidad de León la primera en contar con un Defensor en 1987. Le siguen en antigüedad las de Barcelona y Valencia (Sánchez Giralda, 2009). En el caso español, la creación de la figura se apoya legalmente en el principio de autonomía universitaria, contemplado en la Constitución de 1978. Sin 
embargo, considero que su rápida extensión se debe a la necesidad social de establecer mecanismos de control de los poderes públicos y de defensa de los derechos de los ciudadanos cuando apenas habían pasado diez años desde la muerte del Dictador Franco y ocho años desde las primeras elecciones generales democráticas. Así, en 1996 ya se habían elegido diecisiete defensores o defensoras y se consiguió realizar el primer Encuentro Estatal de Defensores en la Universitat Jaume I.

Añadiré que las Ouvidorias se crearon en Brasil, tal como las primeras Defensorías en España, por decisión de las universidades, después de un largo periodo de dictadura y a partir del principio de autonomía universitaria de la Constitución brasileña de 1988 (Ayoub Richie, 2013), que restauró la democracia. La primera creada fue la de la Universidade Federal do Espírito Santo en 1992.

\section{3. ¿Cómo actúan los Defensores Universitarios?}

El Defensor del Universitario no tiene, salvo algunas excepciones ${ }^{1}$, capacidad ejecutiva y actúa utilizando su autoridad moral o auctoritas para solucionar conflictos, generar buenas prácticas, facilitar consensos, restituir derechos vulnerados o hacer propuestas que contribuyan a un mejor funcionamiento de las universidades. Todas las actuaciones que acabamos de nombrar, redundan en la mejora de la calidad de las universidades. La existencia del Defensor no tiene interés solamente para los miembros de la comunidad universitaria, cuyos derechos protege, sino que interesa a la propia institución.

Una de las características más generales de los defensores universitarios, tanto en el ámbito iberoamericano como en el anglosajón y en el ámbito europeo, es el uso de la mediación como estrategia para resolver conflictos entre personas. La mediación permite que conflictos entre compañeros, entre estudiantes y profesores o entre profesores y autoridades académicas se puedan resolver sin tener que recurrir a procesos judiciales o administrativos que son más lentos, más costosos y que dan lugar a soluciones impuestas. Los conflictos resueltos utilizando la mediación permiten llegar a una solución pactada por las partes en conflicto, asumida por ellas y, por consiguiente, con perspectivas de que la solución sea duradera en el tiempo y de que se genere un clima adecuado para el mejor desarrollo de las tareas encomendadas a los distintos miembros de la comunidad universitaria.

En otros supuestos, el Defensor utiliza su auctoritas para intermediar en una reclamación cuando considera que el reclamante tiene derecho. Así, puede intermediar entre un miembro de la comunidad universitaria y la

1 Por ejemplo, el Independent Adjudicator for Higher Education tiene la capacidad de imponer sanciones a las Universidades inglesas y galesas. 
autoridad ante la que presenta la reclamación. Sirva de ejemplo la reclamación de un estudiante contra un profesor o la de un profesor contra su Decano. En estos supuestos, el Defensor intenta argumentar ante la autoridad la justeza de la reclamación para que sea aceptada en primera instancia o incluso sin que tenga que llegar a presentarse formalmente por el reclamante. Este tipo de actuaciones permiten solucionar muchos problemas con justicia, equidad y agilidad restituyendo la posible vulneración de derechos (Rojo Salgado y Gamero Casado, 2012). En ocasiones, la presentación ante el Defensor de una queja individual o colectiva puede ser un indicio de que algo no funciona bien en la universidad y de que el problema detectado puede afectar a más personas. En esos casos, el Defensor presta un gran servicio a la mejora de la calidad de su universidad recomendando la adopción de medidas, la modificación de normativas o reglamentos o cualquier otra iniciativa que evite que otras personas sufran la misma situación denunciada.

Lo comentado anteriormente forma parte de las múltiples facetas que tiene la actuación de un Defensor Universitario que también puede ejercer funciones de asesoramiento sobre la forma de reclamar un derecho, de información sobre los derechos de cada miembro de la comunidad universitaria, de consejo a las autoridades académicas sobre debilidades o deficiencias del sistema que ha podido percibir desde su posición y un largo repertorio adicional de formas de incidir en la realidad universitaria.

Terminaré esta exposición sobre la forma de actuar del Defensor Universitario con las magistrales palabras del profesor Artur Juncosa en 1996:

Con todo ello, podríamos pasar a describir el perfil de la función del Síndic. Este perfil se inscribiría en un triedro en torno de cuyos ejes y en su espacio definido se desarrollaría dicha función.

1. Más justicia que derecho. Es evidente que el Síndic debe conocer la normativa -o asesorarse a su respecto- y responder de acuerdo con ella. Lo exige su tarea de atender los greuges, los agravios. Pero debe ir, pienso yo, a la raíz de los problemas, analizar si se lesiona la justicia, llamar la atención de los órganos competentes si advierte que las normas, tal vez esclerotizadas, no encauzan ya satisfactoriamente las energías universitarias y llegan incluso a lesionar a los miembros de la comunidad.

2. Más autoridad que poder. La autoridad marca y muestra los fines de la comunidad. El poder, domina los medios. Se ha depositado una confianza en nosotros, la de sugerir soluciones a los organismos universitarios que están al servicio de una institución cuyo fin es la búsqueda de la verdad científica y humana, la transmisión de conocimientos, el desarrollo 
científico y moral de las personas. Este es el horizonte de nuestra tarea, hacia este horizonte estimulamos, en esta tarea se funda nuestra autoridad moral. Por esto recomendamos, sugerimos, a quienes disponen de los medios para que los apliquen con equidad y les advertimos de la responsabilidad ante el Consejo Social u organismo análogo para que este reflexiones sobre la problemática universitaria, sobre su incidencia en las personas, sobre las soluciones que se aplican.

3. Más humanismo que burocracia. Nuestro mundo desarrollado es una selva burocrática tan cruel y peligrosa como la que nos proponen los espectáculos del género de aventuras. Los miembros de la comunidad universitaria son individuos de la especie humana. Las flaquezas humanas pueden enredar en esta selva y a veces herir a los miembros del cuerpo decente y de administración y servicios. Los estudiantes, en particular el que no procede de familias de tradición universitaria y más aún el extranjero no familiarizado con el entramado de nuestra cultura, necesitan de una mano amiga, una acogida fraterna, una actitud para sentirse valorado como hombre comprendido, atendido, informado (Juncosa, 1996).

\section{Características generales del Defensor Universitario en el ámbito Iberoamericano}

Actualmente la figura del Defensor Universitario está muy extendida en México (40 defensorías), Brasil (119), España (70) o Portugal (más de 100)². En el caso de Perú, se ha producido un espectacular aumento del número de Defensorías como consecuencia de la aprobación, en julio de 2014, de una nueva Ley Universitaria que establece en su artículo 133 la creación de Defensorías en las universidades peruanas (LU). Actualmente existen en ese país 66 defensorías (Comunicado SUNEDU, 2017).

La figura del Defensor apenas está implementada en los países más grandes, que cuentan con Defensorías en los siguientes centros: Universidad de Córdoba (Argentina), Universidad Central (Ecuador), Pontificia Universidad Católica y Universidad de Chile (esta última en fase de creación), Universidad Industrial de Santander y Universidad del Atlántico (Colombia) y Universidades Mayor de San Andrés y Autónoma Gabriel René Moreno (Bolivia). También existen Defensorías en países pequeños como es el caso de Costa Rica (Universidad Nacional, Universidad de Costa Rica y UNED, en este caso, solo para estudiantes), Nicaragua (está en fase de creación en la Universidad Nacional Autónoma), Honduras (Universidad Nacional Autó-

2 Información disponible en el sitio web de la Rede de Provedores do Estudante do Ensino Superior http://cms.ua.pt/provedoriasdoestudante/. 
noma), El Salvador (Universidad de Salvador) o Panamá (Universidad de Panamá). Algunas de ellas están asociadas a REDDU.

En general, los defensores universitarios de las universidades iberoamericanas ejercen esa función con carácter temporal y no como una ocupación profesional. En la mayoría de los países, las defensorías son ocupadas por personas que ejercen durante un periodo de tiempo ese servicio para la comunidad universitaria, con una limitación general de 8 años en 2 posibles mandatos. En la mayor parte de los casos, el Defensor debe ser profesor de la propia universidad y reunir algunos requisitos adicionales. No obstante, hay universidades en las que la Defensoría puede ser desempeñada por estudiantes o por personal no académico. También hay algunas universidades en las que el cargo no puede ser desempeñado por profesores en activo y es necesario ser un profesional ajeno a la universidad o un profesor jubilado o emérito3.

Esas características de temporalidad, no profesionalidad y pertenencia a la comunidad universitaria, se pueden también observar en el caso de los médiateurs de las universidades francesas, en las que se está extendiendo rápidamente esa figura. Sin embargo, no es tan general en los ombudsman de las universidades anglosajonas y en las que es frecuente que el cargo está desempeñado por una persona contratada para ese fin y que ejerce la función como profesional (Palazón, 2015). Ese carácter profesional y el hecho de ocuparse, con carácter general, solo de los derechos de los estudiantes hacen que las oficinas de muchos ombudsman anglosajones tengan mucha similitud con las oficinas de atención al usuario (en las universidades públicas) o al cliente (en las universidades privadas).

El Defensor Universitario es independiente de las autoridades académicas y aunque los procedimientos de elección o del nombramiento son diferentes, los defensores son elegidos o nominados por alguna instancia universitaria y una de sus características comunes es la independencia respecto al Rector y a los Órganos de Gobierno.

La Defensoría Universitaria no es, con carácter general, una oficina administrativa de quejas, de atención al usuario o de información, aunque en muchas ocasiones los miembros de la comunidad universitaria acuden a las defensorías para informarse o para pedir asesoramiento, precisamente por la confianza en una instancia que actúa con independencia del resto de la estructura universitaria. Por otra parte, la independencia es fundamental para poder actuar con imparcialidad, que es una característica fundamental del comportamiento de los defensores y que incide en la confianza en sus actuaciones.

Como hemos comentado, los defensores no tienen autoridad ejecutiva y

3 Es el caso de los Síndics de algunas universidades catalanas (Rovira i Virgili, Pompeu Fabra, Politécnica de Catalunya, Autónoma de Barcelona, Lleida). 
su capacidad de incidir en la mejora de sus universidades o de resolver los problemas de quienes acuden a las defensorías depende exclusivamente de su capacidad de argumentación y de persuasión, de su autoridad moral y de su credibilidad.

Los defensores no tienen que atenerse a los procedimientos administrativos, en muchas ocasiones lentos y tediosos, sino que pueden enfrentar los problemas con mucha más agilidad, utilizando el diálogo, la intermediación, los buenos oficios, la empatía, el respeto y el sentido común. En definitiva, se trata de ver personas en lugar de fríos expedientes y hablar con otras personas para buscar soluciones razonables, ágiles y justas a los problemas.

\section{El Defensor Universitario: un factor de buena gobernanza}

Los defensores pueden hacer propuestas para mejorar la calidad de su universidad y esta es en nuestra opinión, una de sus funciones más importantes. En efecto, muchas veces cuando se elaboran las normas en el interior de nuestras universidades, los Órganos de Gobierno intentan contemplar su incidencia en diversos supuestos pero pueden existir otros en los que la aplicación de esa norma puede resultar injusta. En esas circunstancias, se pueden emprender actuaciones de oficio proponiendo modificaciones normativas que permitan evitar situaciones injustas o que ayuden a mejorar la eficiencia y la calidad de una universidad que sea respetuosa con los derechos y la dignidad de los miembros de la comunidad universitaria.

Los defensores, como nos recuerda la cita de Artur Juncosa antes transcrita, nos regimos por el principio de justicia antes que por el de legalidad. En muchas ocasiones nuestra función es velar por que se respeten los derechos que contempla la normativa universitaria, evitando que se produzcan actuaciones arbitrarias. Sin embargo, la aplicación estricta de las normas puede ser injusta en algunos casos concretos. En estos supuestos, solemos atender primero al principio de justicia y actuamos para que se contemplen como excepciones aquellos casos en que la aplicación estricta del principio de legalidad puede ir contra los fines que perseguía quien elaboró la normativa.

Lejos de ser un órgano decorativo e irrelevante, está llamado a cumplir una función fundamental de cara a la buena gobernanza y al buen funcionamiento general de la comunidad universitaria, erigiéndose en esa singular magistratura de opinión; en esa instancia posibilitadora de encuentros y diálogos, constructora de consensos y avenencias, no imponiendo sino buscando siempre elementos que permitan el arreglo y la conciliación y haciendo uso para ello de su auctoritas, prestigio y poder de persuasión. 


\section{Regulación del Defensor Universitario en España}

Como hemos expuesto, la Ley 6/2001 de Universidades no creó la figura del Defensor, sino que se limitó a recoger una realidad existente en muchas universidades, a partir de la propuesta formulada por los Defensores en activo antes de que la figura se hiciera obligatoria. Esto ocurrió en la Disposición adicional decimocuarta de la LOU:

Del Defensor Universitario.

Para velar por el respeto a los derechos y las libertades de los profesores, estudiantes y personal de administración y servicios, ante las actuaciones de los diferentes órganos y servicios universitarios, las Universidades establecerán en su estructura organizativa la figura del Defensor Universitario. Sus actuaciones, siempre dirigidas hacia la mejora de la calidad universitaria en todos sus ámbitos, no estarán sometidas a mandato imperativo de ninguna instancia universitaria y vendrán regidas por los principios de independencia y autonomía (LOU).

Posteriormente, el Estatuto del Estudiante Universitario, de 2010, añadió un apartado en el que se describe la manera de actuar de los defensores (mediación, conciliación, buenos oficios) y su contribución al desarrollo de valores como la convivencia, la cultura de la ética, la corresponsabilidad y las buenas prácticas. También contiene otro apartado en el que se les encarga la tarea de asesoramiento a los estudiantes, tarea que ya estaban realizando por propia iniciativa los defensores:

Artículo 46. El Defensor universitario.

2. Los Defensores Universitarios podrán asumir tareas de mediación, conciliación y buenos oficios, conforme a lo establecido en los Estatutos de las Universidades y en sus disposiciones de desarrollo, promoviendo especialmente la convivencia, la cultura de la ética, la corresponsabilidad y las buenas prácticas.

3. Los Defensores Universitarios asesorarán a los estudiantes sobre los procedimientos administrativos existentes para la formulación de sus reclamaciones, sin perjuicio de las competencias de otros órganos administrativos (REDEEU).

El Defensor normalmente es elegido por el Claustro4, con unos requi-

$4 \quad$ El Claustro Universitario es el máximo órgano de gobierno de la Universidad y está formado por representantes electos de todos los sectores de la comunidad universitaria (profesores, estudiantes y personal no académico). 
sitos de mayoría, que llegan a ser tan exigentes que pueden hacer difícil la elección. Las candidaturas o candidatura pueden ser presentadas, según cada universidad, por el propio candidato, por un número de claustrales, por el Rector o por el Consejo de Gobierno. También está prevista, en algunas universidades, que la elección la haga el Consejo Social o el Consejo de Gobierno.

El Defensor Universitario en España no se somete a mandato imperativo alguno. Es, además, titular de las prerrogativas de inviolabilidad e inmunidad. En las palabras de Argimiro Rojo, Defensor de la Universidad de Vigo, esto significa "que no se le podrán dirigir instrucciones de ninguna autoridad académica, ni tampoco podrá ser expedientado ni sancionado por las opiniones que formule o por los actos que realice en el ejercicio de las competencias propias de su cargo durante su mandato" (Rojo, 2012). Los reglamentos de las Defensorías suelen subrayar la obligación de todos los órganos de gobierno y de todos los miembros de la comunidad universitaria de colaborar con el Defensor en el ejercicio de sus funciones. Además, como también señala acertadamente Argimiro Rojo, el Defensor tiene la capacidad "de llevar a cabo investigaciones completas y exhaustivas", lo cual, según el Defensor de la Universidad de Vigo, "representa, ciertamente, una salvaguardia y garantía en relación a los demandantes" (Rojo, 2012).

Como ya hemos comentado, el Defensor puede emitir recomendaciones sobre un asunto particular, proponiendo una alternativa para solucionarlo. También puede redactar informes de carácter general y de oficio, proponiendo la modificación de la normativa universitaria para resolver determinadas situaciones o para mejorar la calidad del sistema.

El Defensor rinde cuentas de su trabajo ante el órgano que lo eligió generalmente, el Claustro - presentando una Memoria anual que se suele hacer pública en la página web de cada Defensoría. Esa Memoria sirve, además de para dar cuenta de las actuaciones realizadas, para proponer una reflexión sobre los problemas detectados y ofrecer, en su caso, posibles soluciones.

\section{El trabajo en red de las Defensorías}

Las Defensorías españolas se asocian en la Conferencia Estatal de Defensores Universitarios (CEDU). Se trata de una asociación que agrupa a sesenta y dos defensores de universidades públicas y privadas españolas, lo que supone la totalidad de los defensores de las universidades públicas y casi todos los de las privadas. La decisión de crear la CEDU se adoptó en 2007, en el marco del X Encuentro Estatal de Defensores Universitarios. La CEDU celebra anualmente un Encuentro en el que se debate sobre algunos temas propuestos por los defensores y seleccionando los de mayor interés por vo- 
tación entre los socios. En el mismo marco, se celebra la Asamblea anual en la que se tratan asuntos organizativos y se realiza la elección de los cargos de representación y coordinación. También se organizan jornadas técnicas sobre temas de interés común.

Nuestra revista digital, Revista Universidad, Ética y Derechos (Rued@), fue creada como medio de intercambio de reflexiones y opiniones, abierto a todos los miembros de la comunidad universitaria y a las Defensorías iberoamericanas, que ya han empezado a publicar artículos.

Además de los encuentros y asambleas anuales o las jornadas técnicas organizadas por la CEDU, los defensores con algún nexo común también han creado redes y mantienen reuniones en las que se abordan temas de interés. Las redes pueden agrupar a los defensores de una única Comunidad Autónoma (Madrid, Andalucía, Galicia, Castilla y León) o a los defensores de universidades que han constituido una red (Grupo G9 o Xarxa Lluis Vives).

Al margen de los Encuentros anuales, la CEDU cuenta con un método muy útil para realizar consultas sobre cómo actuar ante un problema concreto. El método consiste en algo tan sencillo como plantear la consulta a través de un correo electrónico dirigido a la lista de distribución en la que están todos los defensores o defensoras y el éxito se debe al alto nivel de respuestas y a la rapidez de las mismas. Como ejemplo, los temas consultados por ese procedimiento en el curso 2015/16 han sido los siguientes (entre paréntesis, detrás de cada consulta, está el número de respuestas recibidas a cada consulta):

- Consecuencias del Real Decreto 43/2015, de 2 de febrero, por el que se modifica el Real Decreto. 1393/2007, de 29 de octubre, por el que se establece la ordenación de las enseñanzas universitarias oficiales, y el Real Decreto 99/2011, de 28 de enero, por el que se regulan las enseñanzas oficiales de doctorado (15).

- Acceso a becas de colaboración por doctorandos (11).

- Acceso del Defensor a los expedientes académicos o profesionales (12).

- Aplicación del Real Decreto-ley 14/2012, de 20 de abril, de medidas urgentes de racionalización del gasto público en el ámbito educativo (10).

- Becas en estudios compartidos en dos Campus (3).

- Cobertura de seguro de accidentes del personal de la universidad (12).

- Complemento de productividad del PAS y relación con el de destino (3).

- Grado de consanguineidad de estudiante y profesor (6).

- Plaza de Ayudante Doctor: rechazo de Doctor acreditado por la ANECA con título extranjero homologado por no tener homologada la licen- 
ciatura (7).

- Cotización por desempleo para extranjeros no comunitarios (4).

- Posibilidad de obtener el Diploma de Estudios Avanzados(16).

- Derecho de petición.

- Devolución del precio de matrícula (7).

- Doctorado internacional y estancias fragmentadas (18).

- Normativa de elecciones a Rector (8).

- Exención de tasas de expedición del título por premio extraordinario fin de carrera (10).

- Huelga de estudiantes y evaluación (11).

- Asignaturas incompatibles (13).

- Obligación de impartir clases por videoconferencia (2).

- Petición formulada a través de "Change.org" (3).

- Posibilidad de repetir un examen (7).

- Protocolo de acoso entre iguales (18).

- Protocolo del Defensor (28).

- Pruebas de acceso anteriores a 2008 (6).

- Reducción docente por investigación (10).

- Régimen disciplinario de los estudiantes (9).

- Reserva de plazas en Posgrado para personas con discapacidad (14).

- Suplemento Europeo al Título en Máster y Doctorado (2)

- Sexenios vivos y muertos (6).

- Uso del sello oficial del Departamento (8).

- Validez de la entrega de documentos en oficinas de correos de otros países (3).

Como se puede observar, este procedimiento de consulta es muy ágil y participativo, lo que supone un instrumento de consulta muy útil para quién quiere conocer la opinión de los colegas sobre un problema. Considero que es una de las aportaciones más importantes que la CEDU hace a sus miembros.

En México, seis Defensorías decidieron crear, en 2005, la Red de Defensores, Procuradores y Titulares de Organismos de Defensa de los Derechos Universitarios (REDDU). Es, por lo tanto, una organización mexicana de defensores, de la que son socios, regulares o fundadores, los defensores de veinte universidades mexicanas, y en la que participan, como socios honorarios, quince defensores españoles y defensores de Panamá, Honduras, El Salvador, Perú, Colombia, Brasil, Austria, Estados Unidos, Bélgica y Australia.

Las reuniones de REDDU tienen una faceta organizativa, las Sesiones Ordinarias o Extraordinarias, en la que los socios honorarios (extranjeros) no tienen voto y otra formativa, de debate e intercambio de experiencias que 
se suele traducir en una actividad paralela como los Seminarios de Derechos Universitarios.

De la colaboración de CEDU y REDDU ha surgido la Red Iberoamericana de Defensorías Universitarias (RIDU). En junio de 2015, tras un largo proceso, REDDU y CEDU firmaron un acuerdo para la creación de una Red que favoreciese el intercambio y la colaboración entre las Defensorías existentes en el ámbito iberoamericano y que pudiese ser un instrumento eficaz para impulsar la creación de nuevas Defensorías en ese ámbito. Actualmente, se han inscrito en la RIDU diecisiete Defensorías de España, cinco del Perú, catorce de México, dos de Colombia y una de cada uno de los siguientes países: Bolivia, Brasil, Argentina, Panamá, El Salvador, Chile y Canadá. Considero que estas defensorías constituyen un punto de partida suficiente para que, en breve, la RIDU pueda empezar su andadura.

En Brasil, están muy extendidas las Ouvidorias Universitarias y están asociadas en el Fórum Nacional de Ouvidores Universitários (FNOU), creado en 1999 e integrado por sesenta y una Ouvidorias. Una de las funciones del FNOU es la divulgación de la Institución de la Ouvidoria y el apoyo a las Instituciones de Enseñanza Superior interesadas en crear una Ouvidoria. Según información de la página Web del FNOU, la primera Ouvidoria se creó en 1992 en la Universidade Federal do Espírito Santo. En 1997, solo había cuatro Ouvidorias, pero, en 2001, se habían creado veintiocho y la cifra creció hasta ciento dieciséis en 2011, según la ponencia presentada por la Dra, Cristina Ayoub en el II Seminario sobre Derechos Humanos y Universitarios organizada por REDDU. También en Portugal, existe el Conselho Nacional de Provedores do Estudante do Ensino Superior y la Rede Nacional de Provedores do Estudante de Ensino Superior (REDEPEES). En octubre de 2017 realizaron su VII Encontro.

Los defensores europeos han creado la European Network of Ombudsmen in Higher Education (ENOHE), que suele celebrar un encuentro anual en el que es frecuente la asistencia de defensores de México, Estados Unidos, Canadá, Brasil y Australia, además de los defensores de varios países europeos. Los defensores canadienses se asocian en la Association of Canadian College and University Ombudspersons (ACCUO).

\section{Algunas razones para extender la figura del Defensor Universitario}

La aportación específica del Defensor Universitario a la mejora de la calidad de las universidades, a la defensa de los derechos de sus miembros y a la resolución de conflictos es razón más que suficiente para implantar la figura en aquellas universidades que no disponen de ella y para que las autoridades de los países donde no hay implantación de las Defensorías, o esa implantación es escasa, se planteen una acción decidida para convencer 
a las universidades de la conveniencia de su implantación o para legislar sobre la obligación de implantar esa figura en las universidades. Además, la actuación de los defensores universitarios contribuye a formar transversalmente a nuestros estudiantes como ciudadanos libres y conscientes de la importancia de defender sus derechos y de no comportarse como súbditos. Esa formación transversal de nuestros estudiantes en el respeto de los derechos humanos es, en mi opinión, tan importante como la formación específica para ejercer una profesión. La existencia de elementos de control del ejercicio de la autoridad académica es una contribución muy importante a la mejora de la democracia interna, así como a la transparencia y a la función de servicio público que deben prestar las universidades.

\section{Legislación citada}

LOU

Ley Orgánica 6/2001, de 21 de diciembre, de Universidades, Boletín Oficial del Estado, núm. 307, 24 de diciembre de 2001, pp. 49400-49425.

$\mathrm{LU}$

Ley $\mathrm{N}^{\circ}$ 30220, de 3 de julio de 2014, Universitaria, El Peruano. Diario Oficial, núm.12.914, 9 de julio de 2014, pp. 527211- 527233.

RDEEU

Real Decreto 1791/2010, de 30 de diciembre, por el que se aprueba el Estatuto del Estudiante Universitario, Boletín Oficial del Estado, núm. 318, de 31 de diciembre de 2010, pp. 109353-109380.

\section{Bibliografía citada}

Ayoub Richie, C. (2013). "Los mecanismos de defensa de los derechos universitarios en Brasil". En: Carmona Tinoco, Jorge Ulises (ed.), La vinculación entre los derechos humanos y los derechos universitarios: obra en homenaje al Dr. Jorge Carpizo Mac Grego, México, D.F.: Universidad Nacional Autónoma de México, Defensoría de los Derechos Universitarios. Disponible online: https://biblio.juridicas.unam.mx/bjv/detalle-libro/3988la-vinculacion-entre-los-derechos-humanos-y-los-derechos-universitarios-homenaje-al-dr-jorge-carpizo-mac-gregor\#134471 (último acceso: 1 de diciembre de 2017).

Comunicado SUNEDU. (2017)."66 Universidades cuentan con Defensoría Universitaria". Disponible online: https://www.sunedu.gob.pe/sunedu-66-universidades-cuentancon-defensoria-universitaria/ (último acceso 1 de diciembre de 2017). 
Juncosa, A. (1996). "Reflexiones sobre la figura y función del Síndic de Greuges a partir de una experiencia personal". Comunicación presentada al I Encuentro Estatal de Defensores de la Comunidad Universitaria, Castellón, 19 y 20 de septiembre, 1996. Disponible online: http://cedu.es/images/otros_documentos/conferencias/Juncosa_1996_09. pdf (último acceso: 1 de diciembre de 2017).

Palazón, J. (2015). "Hacia una Red Iberoamericana de Defensores Universitarios", Revista Rued@, o. Disponible online: http://cedu.es/images/rueda/rueda_num_oo.pdf (último acceso: 1 de diciembre de 2017).

SÁnchez Giralda, T. (2010). "El Defensor de la Comunidad Universitaria: una experiencia de privilegio". Comunicación presentada en la jornada conmemorativa del X Aniversario de la implantación de la Institución del Defensor de la Comunidad Universitaria en la Universidad de Valladolid (1999-2009). Disponible online: http://www.cedu.es/ images/otros_documentos/conferencias/Sanchez_2010_02.pdf (último acceso: 1 de diciembre de 2017).

Rojo Salgado, A. (2012). "Una aproximación a la institución del Defensor Universitario: el caso español". Conferencia presentada al Encuentro de la Red de Defensores, Procuradores y Titulares de Organismos de Defensa de los Derecho Universitarios (REDDU), Universidad Autónoma del Estado de Morelos (México). Disponible online: http://www. cedu.es/images/otros_documentos/conferencias/Rojo_2012_10_17.pdf (último acceso 1 de diciembre de 2017).

Rojo Salgado, A. y Gamero CASAdo, E. (Coord.). (2012). "Mesa de trabajo 2. La defensoría universitaria como órgano necesario, tanto más en tiempos de crisis", Conclusiones del XV Encuentro Estatal de Defensores Universitarios, Almería 19 a 21 de septiembre de 2012. Disponible online en: http://cedu.es/images/encuentros/estatales/XVEncuentro/15. mesa2.pdf (último acceso 1 de diciembre de 2017). 